\title{
Design Optimization of Composite Laminated Tube Based on Improved Niching Evolutionary Algorithm
}

\author{
Sen Ma, ${ }^{1}$ Qilin Zhao, ${ }^{2}$ and Darong Pan $^{3}$ \\ ${ }^{1}$ College of Field Engineering, The Army Engineering University of PLA, Nanjing 210007, China \\ ${ }^{2}$ School of Mechanical and Power Engineering, Nanjing Tech University, Nanjing 211800, China \\ ${ }^{3}$ School of Architecture Engineering, Nanjing Institute of Technology, Nanjing 211167, China
}

Correspondence should be addressed to Qilin Zhao; 3442717976@qq.com

Received 21 July 2017; Revised 13 September 2017; Accepted 20 September 2017; Published 13 November 2017

Academic Editor: Francesco Tornabene

Copyright (c) 2017 Sen Ma et al. This is an open access article distributed under the Creative Commons Attribution License, which permits unrestricted use, distribution, and reproduction in any medium, provided the original work is properly cited.

\begin{abstract}
A minimum weight design is developed for a composite laminated tube considering the number of plies as one of the design variables. The objective function is found to be complex, and more than one optimal design point may exist with different numbers of plies. Existing methods based on evolutionary algorithms tend to become trapped around a local optimum and can find no more than one optimal result per calculation. Aiming at the characteristics of the objective function, an improved evolutionary algorithm (INDE for short) is established based on niching technology. The formula for calculating the distance between individuals in the niching technology is improved to satisfy the minimum weight design for the composite laminated tube. As a result, the improved niching evolutionary algorithm offers better global search ability and can find more than one optimal result per calculation for different numbers of plies.
\end{abstract}

\section{Introduction}

Compared with traditional metallic materials, composite materials are anisotropic and designable. These characteristics offer the possibility of obtaining the optimal properties of a composite structure for a specific application, such as the maximum strength, stiffness, or minimum weight [1]. These characteristics also mean that the design of a composite structure involves more design variables and constraints [2], making it more difficult to design a structure with composite materials than with traditional metallic materials.

A common form of composite structures is the composite laminated tube, which is widely used in fields such as oil drilling $[3,4]$, automobile [5-8], aerospace $[9,10]$, and civil engineering [11-13]. Rangaswamy et al. [7] designed automotive drive shafts using E-glass/epoxy laminates and carbon/epoxy laminates. The weights of these drive shafts were $42 \%$ and $87 \%$ lower, respectively, than comparable steel shafts. Zhang et al. [12] explored a novel hybrid FRPaluminum truss system with composite tubes acting as truss bars. The truss system was applied in a bridge, and the transportation performance and assembly performance were improved significantly compared to a steel bridge with the same load capacity.

When laminated composites are used in place of traditional metallic materials for the design of a tube, it is possible to take full advantage of the laminated composites and obtain a tube with better performance and lower weight. However, the type and number of design variables increase significantly. Not only must we determine the design variables that influence the properties of a metallic tube (such as the inner diameter and wall thickness), but we must also determine some typical design variables for laminated composites, such as the ply orientations and number of plies. Intelligent optimization algorithms, especially evolutionary algorithms, are widely used for the optimization of composite laminated tube designs.

Evolutionary algorithms are commonly used for the optimization of composite laminated tubes because they have simple principles and strong applicability. Most applications do not consider the number of plies as a design variable [1418]. Azarafza et al. [18] set the fiber volume ratio, ply orientations, and ply thickness as design variables and performed a multiobjective optimization of the weight and transient 
dynamic response of a composite laminated tube using a basic genetic algorithm (GA). Da Silva et al. [15] optimized the ply orientations and ply thicknesses of composite catenary risers under multiple load cases based on a GA. Previous studies have indicated that evolutionary algorithms are suitable for composite laminated tube optimization with multiple objectives, multiple load cases, or complex constraints under a fixed number of plies. However, the wall thickness of a tube is always controlled by the number of plies because the ply thickness is typically constant for a particular fabrication technology. Some studies considered the number of plies as a design variable when designing composite laminated tubes. An early study by Harrison and Haftka [19] involved optimizing stacking sequences and the number of plies for laminated composite structures based on a GA. "Intron" codes were introduced into the code string to control the number of plies (referred to as the intron method). Todoroki and Sasai [20] used a similar approach. Malott et al. [21] and Rangaswamy and Vijayrangan [22] considered a GA model in which the code string was divided into different parts based on the type of design variable. The number of plies was controlled by a piece of code in the code string (referred to as the segmented coding method).

Previous studies have seldom analyzed the influence of the number of plies on the optimization problem of composite laminated tubes and rarely examine whether existing methods are still applicable. Furthermore, the basic evolutionary algorithm can obtain only one optimal result after a single operation [23], whereas multiple optimal designs may exist in practice. It is extremely important to find all optimal designs because their performance will vary in terms of manufacturing, installation, and maintenance.

In this paper, the number of plies, ply orientations, and inner diameter of a composite laminated tube are considered as the design variables, and the weight is the objective function. First, the influence of the number of plies on the objective function is investigated for a composite laminated tube, and the applicability of existing optimization methods is analyzed. In view of the characteristics of a composite laminated tube considering the number of plies, a niching evolutionary algorithm is proposed for the optimization problem. Finally, test examples are given to verify the accuracy of the analysis and the advantages of the proposed method.

\section{Problem Description}

2.1. Structural Optimization Model. The model described in this paper is a composite laminated tube with height $H$ and inner diameter $d$. The tube is fabricated from $n$ plies, each of thickness $t$. The tube is subjected to load $P$ (Figure 1).

The ply orientation $\theta_{i}(i=1,2, \ldots, n)$ is defined as the angle between the direction of the fiber and the axial direction of the tube.

The optimization problem for minimum weight of a composite laminated tube can be described as follows:

$$
\begin{aligned}
& \text { find } \quad\left(n, \theta_{1}, \theta_{2}, \ldots, \theta_{n}, d\right) \\
& \min \quad m=\rho V=\rho H \pi\left[\left(\frac{d}{2}+n t\right)^{2}-\left(\frac{d}{2}\right)^{2}\right]
\end{aligned}
$$

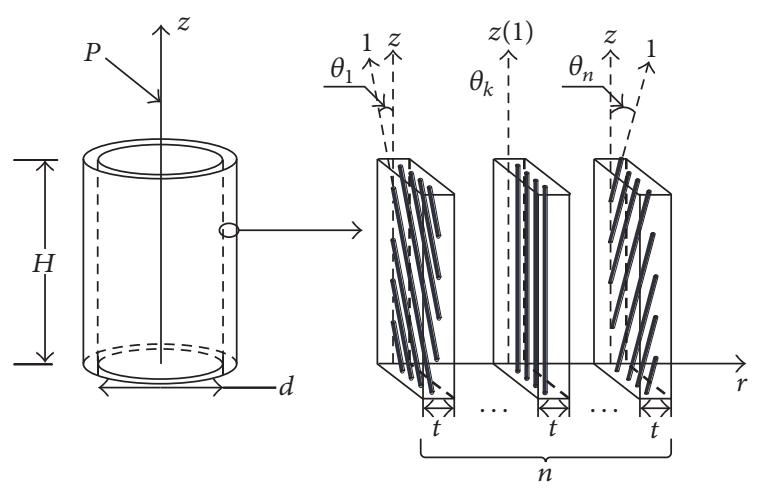

FIGURE 1: Model of the composite laminated tube.

$$
\begin{array}{cc}
\text { s.t. } & g_{1}\left(n, \theta_{1}, \theta_{2}, \ldots, \theta_{n}, d\right) \geq G_{1} \\
& \vdots \\
& g_{\mathrm{nc}}\left(n, \theta_{1}, \theta_{2}, \ldots, \theta_{n}, d\right) \geq G_{\mathrm{nc}} .
\end{array}
$$

In optimization problem (1), design variable $n$ is discrete, $n \in n_{s}$, and $n_{s}=\{1,2, \ldots, N\} . N$ is a fixed upper limit of $n$. $\theta_{i}(i=1,2, \ldots, n)$ is continuous and is typically $\theta_{i} \in \theta_{s}, \theta_{s}=$ $[-90,90] . d$ is continuous, and $d \in d_{s}, d_{s}=\left[d_{l}, d_{u}\right]$, and $d_{l}$ and $d_{u}$ represent the lower and upper limits of $d_{s}$, respectively. $g_{j}(j=1,2, \ldots, \mathrm{nc})$ represents a constraint function such as load capacity, stiffness, or the critical buckling load. $G_{j}(j=$ $1,2, \ldots, \mathrm{nc})$ is the boundary of constraint function $g_{j}$.

2.2. Characteristic Analyses of Optimization Problem. For optimization problem (1), we now analyze the influence of the design variables on the objective function and constraint functions.

As can be seen from formula (1), $m$ is a function related to $n$ and $d$. When $n$ is fixed at $n=k$, the relationship between $m$ and $d$ defines a surface $S_{k}$, which is enclosed by a dashed line in Figure 2 . The value of $n$ determines the number of variables $\theta_{i}(i=1,2, \ldots, n)$. Different values of $n$ correspond to surfaces with different actual dimensions. Normally, constraint function $g_{j}$ is simultaneously related to $n, d$, and $\left(\theta_{1}, \theta_{2}, \ldots, \theta_{n}\right)$, whereas $g_{j}$ is a function of $d$ and $\left(\theta_{1}, \theta_{2}, \ldots, \theta_{n}\right)$ when $n$ is fixed. An individual $I_{i}$ is feasible only when $I_{i}$ satisfies the following conditions:

$$
\begin{gathered}
g_{1}\left(I_{i}\right) \geq G_{1} \\
\vdots \\
g_{\mathrm{nc}}\left(I_{i}\right) \geq G_{\mathrm{nc}} .
\end{gathered}
$$

Therefore, when $n=k$, the local feasible region $F_{k}$ is a collection that belongs to $S_{k}$ (Figure 2). The feasible region $F$ is the union of these incompatible local feasible regions for different numbers of plies:

$$
F=\bigcup_{n=1}^{N} F_{n} .
$$




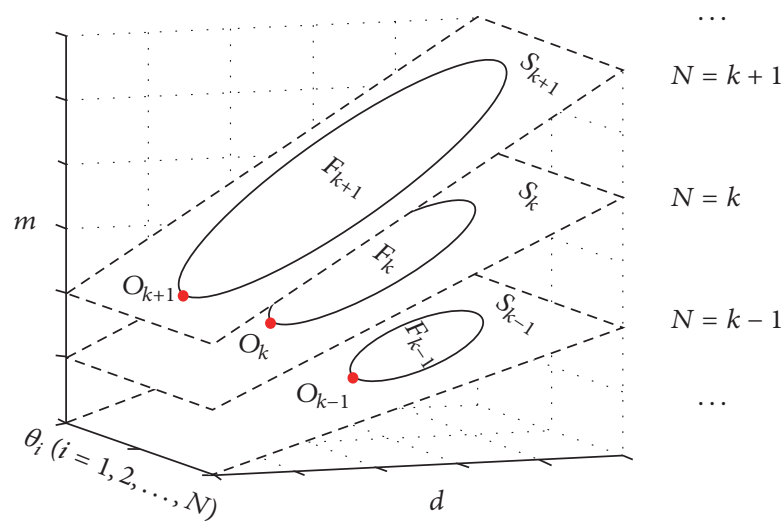

FIGURE 2: Distribution of the objective function.

In local feasible region $F_{k}, m$ and $d$ have a linear relationship, and there is a unique minimum inner diameter $d_{k \text {, min }}$ that minimizes the weight. The design point $O_{k}$ corresponding to $d_{k, \text { min }}$ in $F_{k}$ is a local extreme point of optimization problem (1). Thus, different numbers of plies correspond to different local feasible regions and different local extreme points. The objective function of optimization problem (1) is obviously multimodal in the direction of the number of plies. This phenomenon does not exist if we do not take the number of plies as a variable.

In addition, more than one optimum solution may exist in the set of local extreme points $\left\{O_{k} \mid k=1,2, \ldots, N\right\}$. Taking a case that only considers the axial compression stiffness constraint $g_{s} \geq G_{s}$, for example, the stiffness analysis of the tube is based on the classic lamination theory [24] as a simple approximation method. The axial compression stiffness of the laminated tube can be obtained by adding the axial compression stiffness of each ply together, as

$$
g_{s}=\sum_{i=1}^{n} A_{i} E_{i},
$$

where $A_{i}$ is the cross-sectional area of the $i$ th ply and $E_{i}$ is the effective engineering modulus of the $i$ th ply in the axial direction. The tube has the greatest axial compression stiffness when all plies are axially arranged under this condition [25]; that is, $\theta_{i}=0(i=1,2, \ldots, n)$; the axial compression stiffness can be written as

$$
g_{s}=E_{11} \sum_{i=1}^{n} A_{i} .
$$

$E_{11}$ is the elastic modulus along the fiber orientation. Then, the axial compressive stiffness is proportional to the crosssectional area and there exists a minimum cross-sectional area $A_{\text {min }}$ (or weight) that makes the axial compression stiffness just meet the constraint. The cross-sectional area is a function of number of plies and inner diameter. It can be seen from formula (6) that there exists a unique value of $d$ corresponding to each $n$, solutions for $n$ and $d$ are not unique, any design points $I\left(n, \theta_{1}, \theta_{2}, \ldots, \theta_{n}, d\right)$ that satisfy

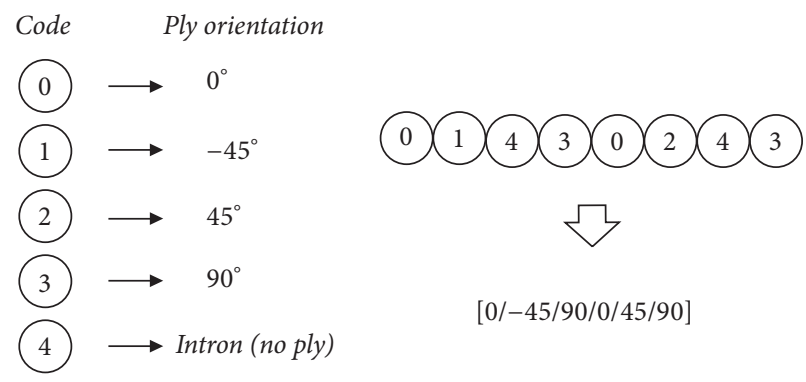

FIgURE 3: Correspondences of codes to actual ply orientations in the intron method.

$$
\begin{aligned}
A_{\text {min }} & =\pi *\left[\left(\frac{d}{2}+n t\right)^{2}-\left(\frac{d}{2}\right)^{2}\right], \\
\theta_{i} & =0 \quad(i=1,2, \ldots, n)
\end{aligned}
$$

are globally optimal solutions, and the optimal solution is not unique.

\subsection{Analysis of the Shortcomings of Existing Method Based} on the Evolutionary Algorithm. Evolutionary algorithms are commonly used to solve optimization problems in composite laminated tubes. The basic evolutionary algorithm is disadvantageous because the process can easily become trapped around a local optimum solution, and the algorithm can only find one extreme point during a single operation. An optimization problem for a composite laminated tube is multimodal, and multiple optimal solutions may exist. This section analyzes whether optimal solutions can be found when basic evolutionary algorithms are used to solve optimization problem (1).

In a basic evolutionary algorithm, the length of the code string depends on the number of design variables and is always fixed during the evolution process. The number of design variables for optimization problem (1) changes with the number of plies. Thus, the length of the code string must be variable, which is impossible in basic evolutionary algorithms. Two optimization methods based on the basic evolutionary algorithm have been proposed to solve this problem. The first is the intron method proposed by Harrison and Haftka [19]. In the intron method, the number of plies is changed by inserting "Intron" codes into the code string. If the length of the code string that represents the ply orientations is $L$, every code in the code string represents one ply with a particular ply orientation or "Intron" ("Intron" means no ply). For example, the search interval for the ply orientation in an optimization problem is $\theta_{s}=\{0, \pm 45,90\}$. Integer codes $0,1,2,3$, and 4 represent ply orientations of $0,45,-45$, 90 , and "Intron," respectively. If the length of the code string is $L=8$, the genotype and phenotype of an individual $I_{i}$ are shown in Figure 3. Codes in the 3rd and 7 th bits have values of 4 , indicating no ply, so the number of plies for individual $I_{i}$ is 6 . The intron method can obtain a variable number of plies on the basis that the length of the code string is constant.

The global search ability of the intron method can be analyzed as follows. $L$ is equal to the upper limit of number 


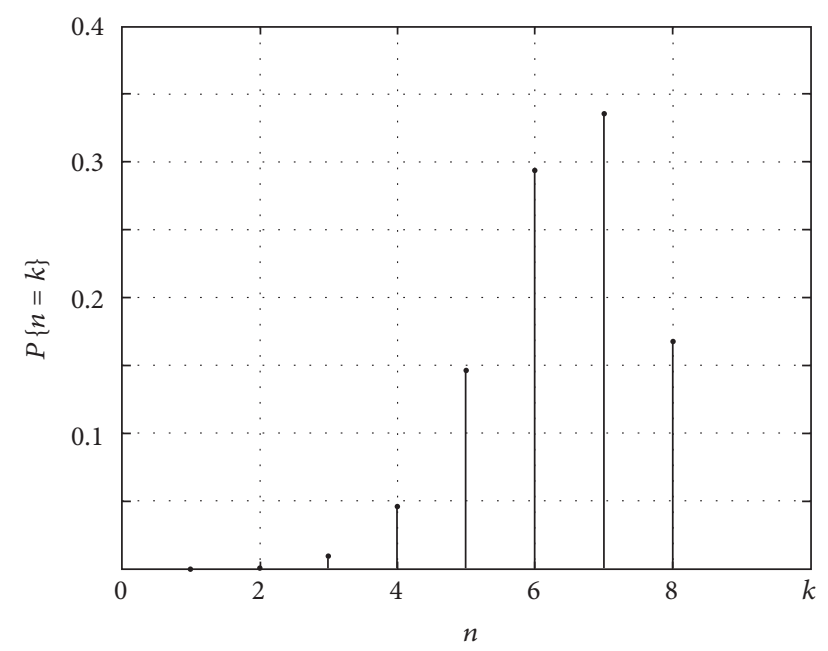

FIgURE 4: Probability distribution of $b(8,0.8)$.

of plies; $N=L$ in other words. At the start of the evolution process, the probability of each code appearing in each bit of the code string is the same, so the probability that "Intron" code 4 appears in any one bit is 0.2 for the example above. Thus, the probability that a code in any bit represents no ply is $p_{s}=0.2$, whereas the probability that a code in any bit represents a physical ply is $p_{b}=1-p_{s}=0.8$. Each code in the code string is random and independent of the other codes in the initial stages of evolution. Therefore, the number of plies for an individual in the population is random and obeys the following binomial distribution:

$$
n \sim b\left(L, p_{b}\right) .
$$

The expected value of $n$ is

$$
E(n)=L * p_{b}
$$

That is, the number of plies for a random individual falls near $E(n)$ with a relatively larger probability. When $p_{b}=0.8, L=8$, $n \sim b(8,0.8)$, and $E(n)=6.4$, as in the example above, the probability that $n=k(k=1, \ldots, N)$ is

$$
p\{n=k\}=\left(\begin{array}{l}
n \\
k
\end{array}\right) p_{b}^{k}\left(1-p_{b}\right)^{(n-k)} .
$$

The probability distribution of $b(8,0.8)$ is shown in Figure 4.

The number of plies for individuals is not uniformly distributed over the entire search interval, particularly for $n_{s}=\{1,2, \ldots, 8\}, p\{n=1\} \approx 0.0001$. This means that it is difficult to generate an individual with exactly 1 ply in the evolutionary process, and the optimization is unlikely to converge to point $O_{1}$ even if this is a global extreme point.

When the search interval for the ply orientation is continuous, the analysis is similar to the above case in which the search interval is discrete. The continuous search interval for ply orientation can be set as $\theta_{s}=[-90, \varphi](\varphi>90)$. When the real code $e_{i}(i=1,2, \ldots, L)$ satisfies the condition that $-90 \leq e_{i} \leq 90$, the code corresponds to a physical ply with a

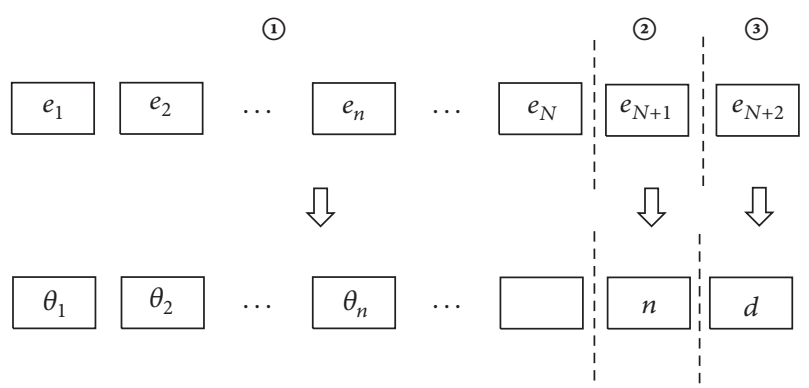

FIGURE 5: Structure of the code string in the segmented coding method.

ply orientation of $\theta=e_{i}$. Otherwise, the code corresponds to no ply, and

$$
p_{b}=\frac{90-(-90)}{\varphi-(-90)}=\frac{180}{\varphi+90} .
$$

$\varphi$ has an impact on $p_{b}$ and affects the distribution of individuals. The uneven distribution of the number of plies negatively affects the convergence of individuals to the global optimal solution.

Another method (based on the basic evolution algorithm) that is frequently used in the optimization of composite laminated structures was proposed by Malott et al. [21] and Rangaswamy and Vijayrangan [22]. Their approach divides the code string into different segments according to the types of design variables, with each segment representing a different type of design variable. This is referred to as the segmented coding method. For optimization problem (1), the code string can be divided into three segments, as shown in Figure 5: Segment (1) represents the $N$ ply orientations; the top $n$ ply orientations are considered to participate in the functions. The value of $n$ is determined by segment (2), and the remaining $n+1 \sim N$ codes are used to keep the length of the code string unchanged. Segment (2) represents the number of plies $n$, and segment (3) represents the inner diameter $d$.

The disadvantages of the segmented coding method for dealing with optimization problem (1) are analyzed below. Also, the case that only the axial compression stiffness constraint $g_{s} \geq G_{s}$ is considered is taken here to illustrate and stiffness analysis of the composite laminated tube is based on the classic lamination theory [24]. A sketch map of the feasible regions of inner diameter $d$ when the numbers of plies are $k$ and $k+1$ is shown in Figure 6. The larger the number of plies (e.g., $n=k+1$ ), the thicker the wall of the tube, and a smaller inner diameter can satisfy the constraint condition. At this point, the minimum inner diameter corresponding to different numbers of plies satisfies

$$
d_{\min , k+1}<d_{\min , k} .
$$

When the ply orientations are all the same, individuals with a larger number of plies are more likely to satisfy the constraint conditions and survive from one generation to the next. Even if the local extreme point $O_{k}$ is the optimum result, individuals can hardly converge to point $O_{k}$ if the value of $d_{u}$ is not appropriate (such as $d_{u}=d_{u_{-}} 1$ in Figure 6(a)), because 


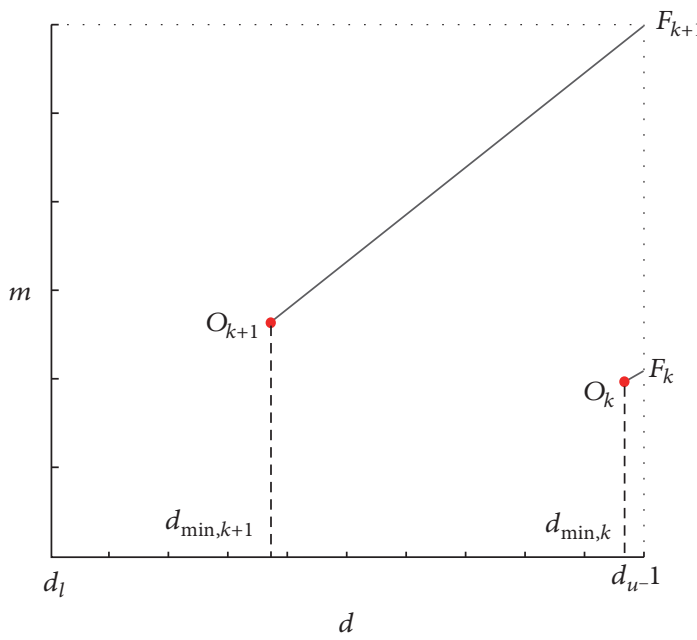

(a) $d_{u}=d_{u-1}$

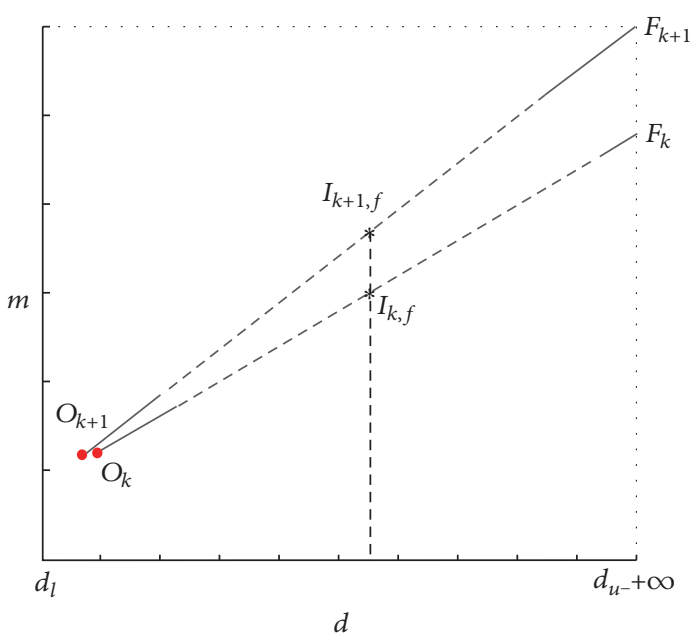

(b) $d_{u}=+\infty$

Figure 6: Effects of $d_{u}$ on $F_{k}$ and $F_{k+1}$.

the corresponding local feasible domain $F_{k}$ is relatively small, and it is difficult to generate individuals within $F_{k}$.

However, when the upper limit of the search interval for inner diameter $d_{u}$ increases, both $F_{k}$ and $F_{k+1}$ increase, $d_{u}=$ $+\infty$ in the extreme case (Figure $6(\mathrm{~b})$ ), and the probability of an individual $I_{k, i}$ satisfying the constraint condition is

$$
\lim _{d_{u} \rightarrow+\infty} p\left(g_{s}\left(I_{k, i}\right)>G_{s}\right)=1 \quad(k=1,2, \ldots, N) .
$$

For any feasible individual $I_{k, f}\left(k, \theta_{1, s}, \theta_{2, s}, \ldots, \theta_{k, s}, d\right)$ with $k$ plies, according to formula (4), when a ply of arbitrary orientation is added to the outer side of tube $I_{k, f}$, the resulting tube $I_{k+1, f}\left(k+1, \theta_{1, s}, \theta_{2, s}, \ldots, \theta_{k, s}, \theta_{k+1, s}, d\right)$ is certain to satisfy constraint condition $g_{s}\left(I_{k+1, f}\right)>G_{s}$. However, the relationship between their objective function values is

$$
m\left(I_{k, f}\right)<m\left(I_{k+1, f}\right) .
$$

When $d_{u} \rightarrow+\infty$,

$$
\begin{aligned}
& \lim _{d_{u} \rightarrow+\infty} F_{k}=S_{k}, \\
& \lim _{d_{u} \rightarrow+\infty} p\left(m\left(I_{k, f}\right)<m\left(I_{k+1, f}\right)\right)=1 \\
&(k=1,2, \ldots, N-1) .
\end{aligned}
$$

Individuals will converge to the local extreme point $O_{k}$ with high probability, even if $\mathrm{O}_{k+1}$ is the optimum result. In this case, individuals easily converge to a local extreme point with a smaller number of plies.

The segmented coding method uses a piece of code string to represent the search interval of inner diameter $d_{s}$. The boundary of $d_{s}$ has an important impact on whether individuals with a particular number of plies meet the constraints, and thereby affects the convergence trend. Sometimes, individuals cannot converge to a global extreme point.

Previous analysis indicates that the optimization problem for a composite laminated tube is typically multimodal in the direction of the number of plies. Existing optimization methods based on the evolution algorithm have shortcomings when solving such problems. It is necessary to find a better optimization method.

\section{Optimization Method}

The probability of an individual evolving to the next generation in a basic evolutionary algorithm is determined merely by its fitness. Individuals easily gather around a local extreme point when the objective function is multimodal, and, typically, only one extreme point can be found. Niching technology is proposed under such a background.

Niching technology divides the individuals in each generation into several categories according to the distances between them. We refer to each category as a niche. Individuals with better fitness are selected from each niche, and these outstanding individuals compose a new group. Crossover and mutation are performed in the new group, and preselection [26], crowding [27], or fitness sharing [28] schemes are used to complete the selection. Take the crowding scheme as an example, the distance (usually the Hamming distance) between every two individuals is calculated before determining which individuals will evolve to the next generation. If the distance is less than the predefined niche radius $R$, the individuals with lower fitness are assigned a fitness near 0 . Individuals that will evolve to the next generation are selected using the adjusted fitness values. From one aspect, the fitness values of worse individuals within a niche are further reduced, and only the optimal individuals within a niche are retained (and will typically evolve to the next generation). Such operations avoid concentrating a large number of individuals. In other respects, if the distances between a special individual and any other individuals are greater than $R$, the special individual will survive. Therefore, individuals in the population will spread throughout the search interval after the adjustment. The two properties 
above have the effect of maintaining individual differences. In theory, the niching evolutionary algorithm has stronger global search ability than the basic evolutionary algorithm. In particular, when the objective function is multimodal, by setting suitable population numbers and niche radii, individuals in the population evolve near all extreme points and eventually converge to these extrema. Thus, all optimal solutions can be found.

3.1. Improved Niching Evolutionary Algorithm. The implementation of niching technology is now described. Before the evolution of current individuals to the next generation, the distance between any two individuals $I_{i}\left(x_{i, 1}, x_{i, 2}, \ldots, x_{i, v}\right)$ and $I_{j}\left(x_{j, 1}, x_{j, 2}, \ldots, x_{j, v}\right)$ is calculated as

$$
D_{i j}=\left\|I_{i}-I_{j}\right\|=\left(\sum_{k=1}^{v}\left(x_{i, k}-x_{j, k}\right)\right)^{1 / 2},
$$

where $v$ is the number of design variables. We deem individuals $I_{i}$ and $I_{j}$ to be in the same niche when $D_{i j} \leq R$ ( $R$ is the predefined niche radius). The fitness values of $I_{i}$ and $I_{j}$ are then compared, and a penalty is imposed on the individual with the smaller fitness:

$$
f_{\min \left(I_{i}, I_{j}\right)}=\text { Penalty, }
$$

where the value of "Penalty" is typically close to zero.

From previous analyses of optimization problem (1), we know that the number of design variables varies with the number of plies and that it is difficult to calculate the distances between individuals using formula (15). In view of the characteristics of the objective function in terms of the number of plies, there are local extreme points corresponding to each ply number. If each number of plies is seen as a niche, evolution will occur among individuals of all numbers of plies, and the phenomenon in which individuals converge to the local extreme point of a special number of plies is avoided. Thus, when calculating the distance between any two individuals $I_{i}, I_{j}$ in problem (1), only the distance component in the direction of the number of plies is calculated:

$$
D_{i j}=n_{i}-n_{j}
$$

where $n_{i}$ represents the number of plies of individual $I_{i}$.

We believe $I_{i}$ and $I_{j}$ are in the same niche if $D_{i j}=0$ (i.e., the numbers of plies for $I_{i}$ and $I_{j}$ are the same). The fitness values of $I_{i}$ and $I_{j}$ are then compared, and a penalty is imposed on the individual with the smaller fitness. We refer to this as the effective direction niching technique.

Niching technology is a selection strategy that is commonly used in conjunction with an evolutionary algorithm. In this paper, the effective direction niching technique is combined with the differential evolution (DE) algorithm proposed by Storn and Price in 1997 [29] to solve optimization problem (1). The optimization method proposed here is simplified as the INDE method.

3.2. Process of INDE. The main difference between the INDE method and existing methods is that the fitness values of individuals should be adjusted before the selection operations. Before optimization, the following steps should be performed.

(1) Encoding Mode. The search interval contains the number of plies $n_{s}=\{1,2, \ldots, N\}$, inner diameter $d_{s}=\left[d_{l}, d_{u}\right]$, and ply orientation $\theta_{s}=[-90,90]$. The structure of the real code string is shown in Figure 5, and the length is $N+2$. The range of the real code is set as $[-90,90]$; the purpose of this is to directly equate code values with ply orientations. The first $N$ codes $e_{1} \sim e_{n}$ represent ply orientations, whereas the $(N+1)$ th code $e_{N+1}$ represents the number of plies. The relationship between them is

$$
= \begin{cases}N & \text { if } e_{N+1}=90 \\ \text { Round }\left(\frac{N *\left(e_{N+1}+90\right)}{180}+0.5\right) & \text { else, }\end{cases}
$$

where the function "Round" applies the rounding operation to the argument. The $(N+2)$ th code $e_{N+2}$ represents the inner diameter, given by

$$
d=\frac{\left(e_{N+2}+90\right)\left(d_{u}-d_{l}\right)}{180}+d_{l} .
$$

(2) Transformation of the Objective Function. The penalty function method proposed by Rajeev and Krishnamoorthy [30] is adopted to transform the constrained optimization into an unconstrained form. If an individual satisfies the constraints, its objective function value remains unchanged. If the individual does not satisfy the constraints, the objective function value of the individual increases sharply, significantly reducing its fitness. Take the axial compression stiffness constraint $g_{s} \geq G_{s}$ as an example. The axial compression stiffness of individual $I_{i}$ is $g_{s}\left(I_{i}\right)$, and the axial compression stiffness violation-factor $c_{s}$ is defined as

$$
c_{s}= \begin{cases}0 & \text { if } g_{s}\left(I_{i}\right) \geq G_{s} \\ \frac{G_{s}}{g_{s}\left(I_{i}\right)}-1 & \text { else. }\end{cases}
$$

Other violation-factors are defined in a similar manner to $c_{s}$. The relationship between the different violation-factors is

$$
C=\sum_{i=1}^{\mathrm{nc}} c_{i}
$$

Finally, the objective function $m$ is transformed into the following form:

$$
\phi\left(I_{i}\right)=m\left(I_{i}\right)\left(1+K_{c} * C\right),
$$

where $\phi$ represents the converted objective function and $K_{c}$ is a constant used to control punishment (typically, $K_{c}=10$ ).

\section{(3) Process of INDE in Solving Optimization Problem (1)}

Step 1. Generate the initial population op of $M$ individuals and calculate the fitness of each individual. The size of $M$ is typically 10 20 times the number of niches. The number of niches is the same as $N$, so $M$ is $10 \sim 20$ times $N$. 
TABLE 1: Material properties of E-glass/epoxy laminate.

\begin{tabular}{lc}
\hline Material properties & Value \\
\hline$E_{1} / \mathrm{GPa}$ & 95 \\
$E_{2}=E_{3} / \mathrm{GPa}$ & 7.4 \\
$G_{12}=G_{21} / \mathrm{GPa}$ & 3.6 \\
$v_{12}=v_{13}$ & 0.3 \\
$v_{23}$ & 0.35 \\
$X_{t} / \mathrm{MPa}$ & 1100 \\
$X_{c} / \mathrm{MPa}$ & 750 \\
$Y_{t}=Y_{c} / \mathrm{MPa}$ & 31 \\
$Z_{t}=Z_{c} / \mathrm{MPa}$ & 110 \\
$S_{x y} / \mathrm{MPa}$ & 49 \\
\hline
\end{tabular}

Step 2. Arrange the individuals of $o p$ in descending order according to their fitness values. Store the top $T$ individuals to form the crowding population $n p$ (the size of $n p$ is always $M / 2$ ). The role of $n p$ is to concentrate population op 2 in Step 4 to retain good genes.

Step 3 (replication). Individuals in $n p$ are replicated in proportion to their fitness values. Population op1 of $M$ individuals is obtained. Replication can achieve the goal of retaining good codes.

Step 4. Perform crossover, mutation, and selection operations on population $o p 1$. Population $o p 2$ is thereby obtained.

Step 5 (niching operation). Merge $n p$ into op 2 to obtain population $n p 3$ of $(M+T)$ individuals. Adjust the fitness values of individuals in population $n p 3$ according to formula (17) to obtain population $n p 4$. Arrange the individuals of $o p$ in descending order according to their fitness values. Store the top $M$ individuals to form the new $o p$ and the top $T$ individuals to form the new $n p$.

Step 6 (termination). If the termination condition is satisfied, stop and output the result. Otherwise, go to Step 2.

\section{Test Example and Discussion}

\subsection{Test Example 1}

4.1.1. Description of the Test Example. The length of a cylindrical truss rod is $H=100 \mathrm{~mm}$. The rod is fabricated from E-glass/epoxy laminates of thickness $t=0.05 \mathrm{~mm}$ and density $\rho$; the tube is subjected to axial compression load $P$. The laminates are balanced and symmetrically arranged. The properties of the E-glass/epoxy laminate are listed in Table 1.

The strength constraint $g_{\text {st }} \geq G_{\text {st }}$ and local buckling critical load constraint $g_{\mathrm{bl}} \geq G_{\mathrm{bl}}$ are considered, where $G_{\mathrm{st}}=$ $2.0 e^{5} \mathrm{~N}$ and $G_{\mathrm{bl}}=2.0 e^{5} \mathrm{~N}$. The strength calculation is based on the 3D elastic theory [31], first ply failure criteria, and Tsai and $\mathrm{Wu}$ tensor theory [32]. The calculation of the local buckling critical load is based on classical lamination theory [33].

First, the intron method and segmented coding method (based on the basic DE algorithm) are used to solve the
TABLE 2: Parameters of DE.

\begin{tabular}{lc}
\hline Parameter & Value \\
\hline Population size & 200 \\
Maximum generations & 2500 \\
Crossover probability & 0.9 \\
Mutation probability & 0.5 \\
\hline
\end{tabular}

TABLE 3: Effects of $\varphi$ on results of intron method.

\begin{tabular}{lcccccc}
\hline$\varphi$ & 500 & 400 & 300 & 200 & 100 & 92 \\
\hline$p_{b}$ & 0.305 & 0.367 & 0.462 & 0.621 & 0.947 & 0.989 \\
$E(n)(\times 4)$ & 4.27 & 5.14 & 6.47 & 8.70 & 13.26 & 13.85 \\
$m_{\text {opt }}(\rho H)$ & 418.1 & 346.4 & 346.9 & 296.0 & 294.1 & 268.6 \\
$n_{\text {opt }}(\times 4)$ & 7 & 8 & 8 & 9 & 9 & 10 \\
\hline
\end{tabular}

problem in the test example. The results are presented to verify the analysis in Section 2. The problems are then solved using the proposed INDE method. The effects of DE and the INDE method are compared. The parameters for DE are listed in Table 2.

\subsubsection{Discussion}

(1) Intron Method. The parameters of the intron method based on the basic DE algorithm are listed in Table 2. The upper limit of the number of plies is set as $N=14, d_{s}=$ $[5 \mathrm{~mm}, 60 \mathrm{~mm}], \theta_{s}=[-90,90]$, and $e_{i} \in[-90, \varphi](\varphi>90$, $i=1,2, \ldots, N)$. The binomial distribution probabilities $p_{b}$ obtained according to formula (10) are presented in the second row of Table 3 for different $\varphi$ values. The expected values for ply numbers $E(n)$ obtained according to formula (8) are presented in the third row of the table. The numbers in the fourth and fifth rows are the optimal results and corresponding numbers of plies for different $\varphi$ values.

The results indicate that the expected values for each ply number and the optimal results vary with changes in $\varphi$. The results are optimal when $\varphi=92$, corresponding to 10 plies. Other values of $\varphi$ give greater objective function values, and the corresponding ply numbers are different. Hence, the value of $\varphi$ in the intron method has an important influence on the optimization results. An improper $\varphi$ value may cause the results to converge to a local extreme point. The previous analysis of this method is verified.

(2) Segmented Coding Method. In this section, we examine the impact of the search interval $\left[d_{l}, d_{u}\right]$ on the optimization results given by the segmented coding method. The parameters of the segmented method (based on the basic DE algorithm) are listed in Table 2. The structure of the code string is the same as that in Figure 5. The upper limit on the number of plies is set to $N=14$ and $d_{l}$ is fixed at $d_{l}=5 \mathrm{~mm}$. The optimal results and numbers of plies corresponding to different $d_{u}$ values are listed in the second and third rows of Table 4 .

When $d_{u}$ is set to 20,25 , or 40 , the optimization results are relatively good. For other values of $d_{u}$, the objective function values are clearly higher, and the corresponding 
TABLE 4: Effects of $d_{u}$ on results of segmented coding method.

\begin{tabular}{lccccccc}
\hline$d_{u}(\mathrm{~mm})$ & 20 & 25 & 40 & 50 & 60 & 80 & 120 \\
\hline$m_{\text {opt }}(\rho H)$ & 268.8 & 267.5 & 268.9 & 294.1 & 352.9 & 359.2 & 398.2 \\
$n_{\text {opt }}(\times 4)$ & 11 & 10 & 10 & 9 & 8 & 8 & 7 \\
\hline
\end{tabular}

TABLE 5: Results of the INDE method $(\rho H)$.

\begin{tabular}{lccccc}
\hline$n_{\mathrm{opt}}(\times 4)$ & \multicolumn{5}{c}{$d_{u}(\mathrm{~mm})$} \\
\hline 7 & 20 & 40 & 60 & 80 & 120 \\
8 & - & - & 392.5 & 439.9 & 423.8 \\
9 & - & 356.1 & 360.4 & 363.9 & 357.6 \\
10 & - & 297.8 & 301.9 & 302.3 & 297.2 \\
11 & - & 268.8 & 267.3 & 270.9 & 269.8 \\
12 & 267.2 & 269.5 & 269.4 & 270.6 & 269.2 \\
13 & 275.6 & 273.7 & 273.7 & 269.4 & 269.7 \\
14 & 268.8 & 278.2 & 275.6 & 275.5 & 273.0 \\
\hline
\end{tabular}

ply numbers are different. The results in Table 4 show that the value of $d_{u}$ greatly affects the optimization results in the segmented coding method. An improper value of $d_{s}$ may lead to convergence to a local extreme point. The previous analysis of the segmented coding method is verified.

(3) INDE Method. The INDE method is now used to optimize the test example. The upper limit of the number of plies is set as $N=14$, and $d_{l}$ is fixed at $d_{l}=5 \mathrm{~mm}$. The structure of the code string is the same as in Figure 5. Results corresponding to different $d_{u}$ values are listed in Table 5 .

The results for the different methods indicate that the intron technology and segmented coding method based on the basic evolutionary algorithm can only produce optimal results once per calculation. Moreover, the optimal results are strongly affected by parameters in the optimization method, such that they sometimes cannot converge to global extreme points. The INDE method can produce more than one optimal result for different inner diameters. At the same time, the results are less affected by the parameters in the optimization model. This example demonstrates the advantages of the proposed INDE method.

4.2. Test Example 2. Rangaswamy et al. [7] conducted the optimization of a tubular drive shaft made of E-glass/epoxy laminates based on the basic GA. Weight $(m)$ of the drive shaft was set to be the objective function. The design variables included the number of plies $n$, thickness of the ply $t$, and ply orientations $\theta_{i}$. The design of the drive shaft needed to meet the following performance requirements: the torque transmission capability $(T)$ and torsional buckling capacity $\left(T_{c r}\right)$ of the drive shaft should be larger than $3500 \mathrm{Nm}$ and the fundamental natural bending frequency $\left(N_{c r t}\right)$ should be higher than $6500 \mathrm{rpm}$. The performance calculations are based on the classical lamination theory [24]; more details can be allocated in [7]. Optimal design values based on GA in the literature are shown in Table 6. Also, the problem is solved based on INDE proposed by the paper; number of individuals in the population, crossover probability, and mutation probability are shown in Table 2. After 1000 times of iterations, parts of the optimal design values of INDE are shown in Table 6 to compare with results of GA.

From Table 6, the optimal design value of composite drive shaft based on GA is $4.44 \mathrm{~kg}$, multiple better design values of different numbers of plies can be obtained based on INDE, and the optimal design value is $3.54 \mathrm{~kg}$, decreased by $20.27 \%$ compared to that of GA. The advantages of the INDE method are demonstrated again.

\section{Conclusions}

Through the research and analysis in this study, some useful results have been obtained. For the optimization problem of a composite laminated tube, considering the number of plies as one of the design variables, there is at least one local extreme point for each number of plies and more than one optimal design point. The distribution of the objective function is more complicated than that when the number of plies is fixed. While dealing with such problems, existing models based on the basic evolutionary algorithm have disadvantages. The results are easily affected by the initial parameters and not all optimal design points can be found. The proposed INDE method combines the advantages of the niching evolutionary algorithm and the characteristics of the objective function. Test examples have verified the analyses of the performance of existing methods based on the basic evolutionary algorithm and the INDE method. The calculation results show that the INDE method has stronger global search ability and can find more than one optimal result for different numbers of plies.

\section{Nomenclature}

$A_{\text {min }}$ : Cross-sectional area corresponding to the minimum weight of the composite laminated tube

$A_{i}$ : $\quad$ Cross-sectional area of the $i$ th ply

C: $\quad$ Violation-factor of constraints

$c_{s}$ : Violation-factor corresponding to constraint function $g_{s}$

$D_{i j}$ : Distance between individuals $I_{i}$ and $I_{j}$

$d$ : Inner diameter of the composite laminated tube

$d_{s}: \quad$ Search interval of $d$

$d_{l}$ : $\quad$ Upper limit of $d_{s}$

$d_{u}: \quad$ Lower limit of $d_{s}$

$d_{k, \text { min }}$ : The minimum inner diameter in local feasible region when $n=k\left(F_{k}\right)$

$E(n)$ : Expected value of number of plies $n$

$E_{11}$ : The elastic modulus along the fiber orientation

$E_{i}$ : The effective engineering modulus in the axial direction of the $i$ th ply

$e_{i}: \quad$ The $i$ th code in the code string

$F$ : Feasible region

$F_{k}$ : Local feasible region when $n=k$

$f_{I}: \quad$ Fitness of individual $I$

$G_{j}: \quad$ Boundary of the $j$ th constraint function $g_{j}$

$g_{j}: \quad$ The $j$ th constraint function

$H$ : Height of the composite laminated tube

$I_{i}: \quad$ A random individual

$I_{k, i}: \quad$ A random individual with the number of pliers of $k$ 


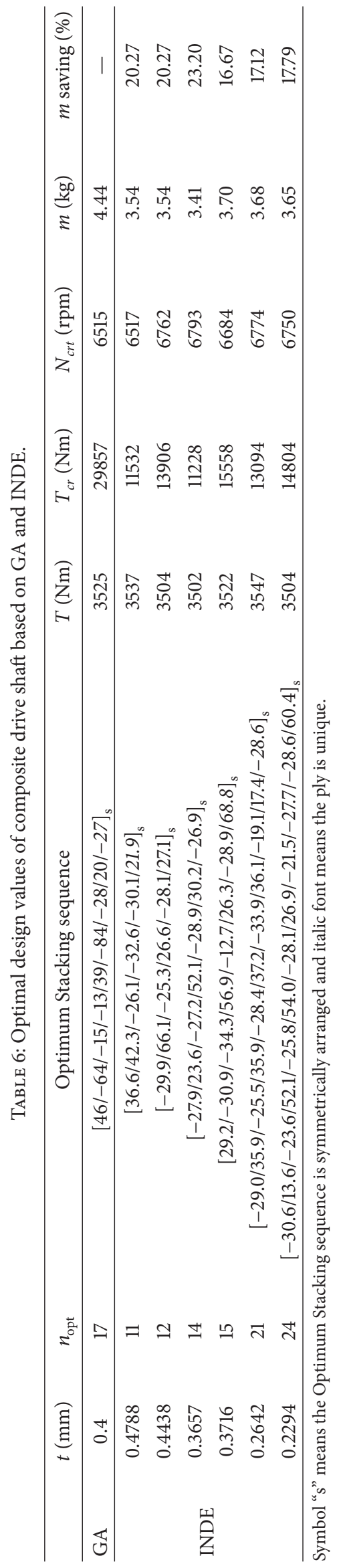


$I_{k, s}:$ A random individual within the feasible region of number of pliers of $k$

$K_{c}$ : Factor used to control the power of punishment

$L$ : Length of the code string that represents the ply orientations

$M$ : Number of individuals in populations

$m$ : Weight of the tube

$N$ : Upper limit of search interval of $n_{s}$

$n$ : Number of plies of the composite laminated tube

$n_{s}$ : Search interval of variable $n$

$n_{x}$ : Number of plies of individual $x$

nc: Number of constraint functions

$O_{k}$ : Design point corresponding to $d_{k, \min }$

$P$ : Compressive load applied at the tube centroid

$p_{b}$ : Probability that a code in the code string represents a ply

$p_{s}$ : Probability that a code in the code string represents no ply

$R:$ The niche radius

$S_{k}$ : Relationship between $m$ and $d$ when $n$ is fixed at $n=k$

$t$ : Thickness of the ply

$V$ : Volume of the composite laminated tube

$v$ : Number of design variables

$\theta_{i}$ : Ply orientation of the $i$ th laminate

$\rho$ : Density of the laminate

$\theta_{s}$ : Search interval of ply orientation

$\varphi$ : Upper limit of $\theta_{s}$ in the intron method

$\phi$ : The converted objective function.

\section{Conflicts of Interest}

The authors declare that there are no conflicts of interest regarding the publication of this paper.

\section{Acknowledgments}

This work is supported by the National Natural Science Foundation of China (Grant no. 11372355 and Grant no. 51708552) and the Natural Science Foundations of Jiangsu Province, China (Grant no. BK20170752).

\section{References}

[1] M. Walker and R. E. Smith, "A technique for the multiobjective optimisation of laminated composite structures using genetic algorithms and finite element analysis," Composite Structures, vol. 62, no. 1, pp. 123-128, 2003.

[2] B. Paluch, M. Grédiac, and A. Faye, "Combining a finite element programme and a genetic algorithm to optimize composite structures with variable thickness," Composite Structures, vol. 83, no. 3, pp. 284-294, 2008.

[3] M. M. Salama, G. Stjern, T. Storhaug, B. Spencer, and A. Echtermeyer, "The First Offshore Field Installation for a Composite Riser Joint," in Proceedings of the Offshore Technology Conference, pp. 247-253, usa, May 2002.

[4] M. Salama and B. Spencer, Multiple seal design for composite risers and tubing for offshore applications, U.S. Patent, No. 6719058, 2004.
[5] M. M. Shokrieh, A. Hasani, and L. B. Lessard, "Shear buckling of a composite drive shaft under torsion," Composite Structures, vol. 64, no. 1, pp. 63-69, 2004.

[6] A. R. Abu Talib, A. Ali, M. A. Badie, N. Azida Che Lah, and A. F. Golestaneh, "Developing a hybrid, carbon/glass fiberreinforced, epoxy composite automotive drive shaft," Materials and Corrosion, vol. 31, no. 1, pp. 514-521, 2010.

[7] T. Rangaswamy, S. Vijayarangan, R. A. Chandrashekar, T. K. Venkatesh, and K. Anantharaman, "Optimal design and analysis of automotive composite drive shaft," in Proceedings of the International Symposium of Research Students on Materials Science and Engineering, vol. 4, pp. 1-9, 2002.

[8] H. Ghoneim and D. J. Lawrie, "Analysis of the flexural vibration of a composite drive shaft with partial cylindrical constrained layer damping treatment," Journal of Vibration and Control, vol. 12, no. 1, pp. 25-55, 2006.

[9] D. C. Jegley, K. C. Wu, J. E. Phelps, M. J. Mckenney, and L. Oremont, "Structural efficiency of composite struts for aerospace applications," Journal of Spacecraft and Rockets, vol. 49, no. 5, pp. 915-924, 2012.

[10] J. Smith, "Evolved composite structures for Atlas V," in Proceedings of the 38th AIAA/ASME/SAE/ASEE Joint Propulsion Conference Exhibit, Indianapolis, Ind, USA, July 2002.

[11] D. Zhang, Y. Huang, Q. Zhao, F. Li, F. Li, and Y. Gao, "Structural performance of a hybrid FRP-aluminum modular triangular truss system subjected to various loading conditions," The Scientific World Journal, vol. 2014, Article ID 615927, 2014.

[12] D. Zhang, Q. Zhao, Y. Huang, F. Li, H. Chen, and D. Miao, "Flexural properties of a lightweight hybrid FRP-aluminum modular space truss bridge system," Composite Structures, vol. 108, no. 1, pp. 600-615, 2014.

[13] X. Yang, Y. Bai, and F. Ding, "Structural performance of a largescale space frame assembled using pultruded GFRP composites," Composite Structures, vol. 133, pp. 986-996, 2015.

[14] A. Todoroki and M. Sasai, "Improvement of design reliability for buckling load maximization of composite cylinder using genetic algorithm with recessive-gene-like repair," JSME International Journal, Series A: Solid Mechanics and Material Engineering, vol. 42, no. 4, pp. 530-536, 1999.

[15] R. F. Da Silva, F. A. F. Teófilo, E. Parente Jr., A. M. C. D. Melo, and Á. S. D. Holanda, "Optimization of composite catenary risers," Marine Structures, vol. 33, pp. 1-20, 2013.

[16] T. Messager, M. Pyrz, B. Gineste, and P. Chauchot, "Optimal laminations of thin underwater composite cylindrical vessels," Composite Structures, vol. 58, no. 4, pp. 529-537, 2002.

[17] P. Y. Tabakov and E. B. Summers, "Layup optimization of multilayered anisotropic cylinders based on a 3D elasticity solution," Computers \& Structures, vol. 84, no. 5, pp. 374-384, 2006.

[18] R. Azarafza, S. M. R. Khalili, A. A. Jafari, and A. Davar, "Analysis and optimization of laminated composite circular cylindrical shell subjected to compressive axial and transverse transient dynamic loads," Thin-Walled Structures, vol. 47, no. 8-9, pp. 970983, 2009.

[19] P. Harrison and R. Haftka, "Design of stiffened composite panels by genetic algorithm and response surface approximations," in Proceedings of the 36th Structures, Structural Dynamics and Materials Conference, pp. 58-68, New Orleans, LA, USA, April 1995.

[20] A. Todoroki and M. Sasai, "Optimizations of stacking sequence and number of plies for laminated cylinders using GA with 
intron genes," Advanced Composite Materials: The Official Journal of the Japan Society of Composite Materials, vol. 12, no. 4, pp. 331-343, 2004.

[21] B. Malott, R. C. Averill, E. D. Goodman, Y. Ding, and W. F. Punch, "Use of genetic algorithms for optimal design of laminated composite sandwich panels with bending-twisting coupling," in Proceedings of the 37th AIAA/ASME/ASCE/AHS/ASC Structure, Structural Dynamics and Materials Conference, pp. 1574-1581, Salt Lake City, Utah, USA, April 1996.

[22] T. Rangaswamy and S. Vijayrangan, "Optimal sizing and stacking sequence of composite drive shafts," Materials science, vol. 11, no. 2, pp. 133-139, 2005.

[23] M. Hall, "A Cumulative Multi-Niching Genetic Algorithm for Multimodal Function Optimization," International Journal of Advanced Research in Artificial Intelligence, vol. 1, no. 9, pp. 6-13, 2012.

[24] E. Reissner, "On generalized two-dimensional plate theoryI," in Proceedings of the On generalized two-dimensional plate theory-I, vol. 5, pp. 525-532, 1969.

[25] J. R. Vinson and R. L. Sierakowski, The behavior of structures composed of composite materials, Springer Science Business Media, Dordrecht, The Netherlands, 2012.

[26] Cavicchio. and D. Joseph, Adaptive search using simulated evolution [Ph.D. thesis], University of Michigan, 1970.

[27] D. Jong and A. Kenneth, An analysis of the behavior of a class of genetic adaptive systems [Ph.D. thesis], University of Michigan, 1975.

[28] D. E. Goldberg and J. Richardson, "Genetic algorithms with sharing for multimodal function optimization," in Proceedings of the Second International Conference on Genetic Algorithms, pp. 41-49, 1987.

[29] R. Storn and K. Price, "Differential Evolution - A Simple and Efficient Heuristic for Global Optimization over Continuous Spaces," Journal of Global Optimization, vol. 11, no. 4, pp. 341359, 1997.

[30] S. Rajeev and C. S. Krishnamoorthy, "Discrete optimization of structures using genetic algorithms," Journal of Structural Engineering, vol. 118, no. 5, pp. 1233-1250, 1992.

[31] S. G. Lekhnitskii, Theory of elasticity of an anisotropic elastic body, Mir Publishers, Moscow, Russia, 1963.

[32] S. W. Tsai and E. M. Wu, "A general theory of strength for anisotropic materials," Journal of Composite Materials, vol. 5, pp. 58-80, 1971.

[33] V. I. Weingarten, P. Seide, and J. P. Peterson, Buckling of thinwalled circular cylinders NASA Space Vehicle Design Criteria, National Aeronautics and Space Administration, No. NASA SP-8007, 1968. 


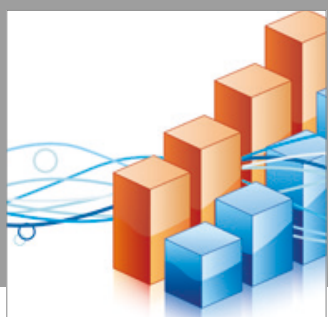

Advances in

Operations Research

vatersals

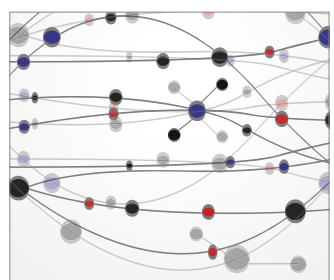

\section{The Scientific} World Journal
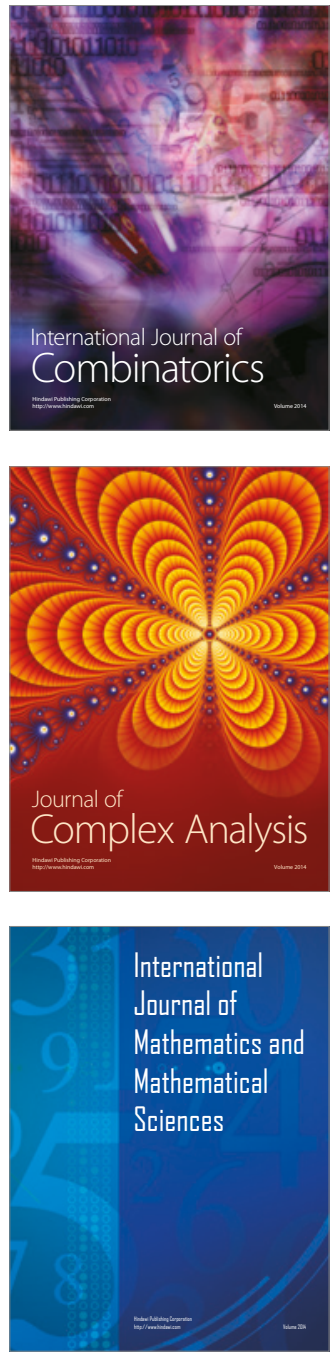
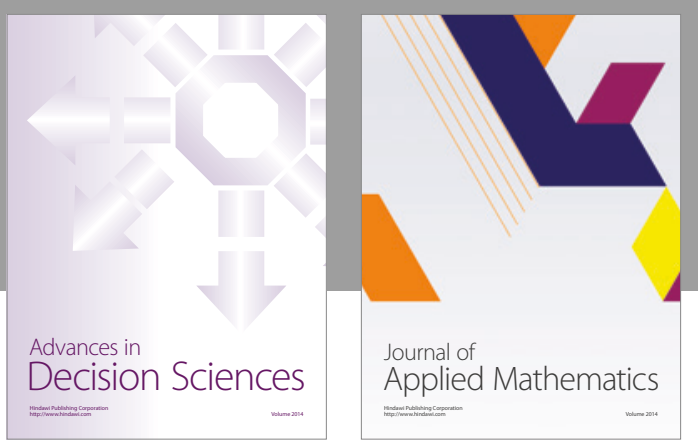

Algebra

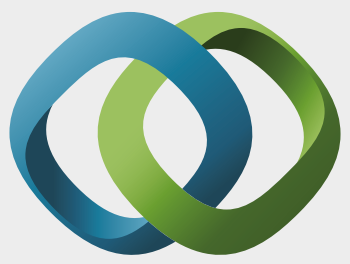

\section{Hindawi}

Submit your manuscripts at

https://www.hindawi.com
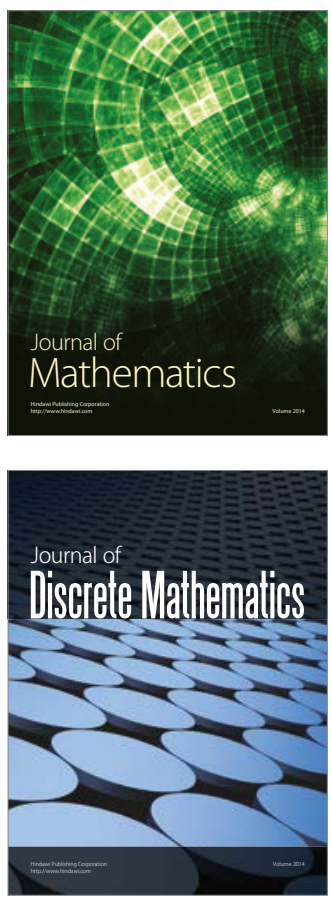

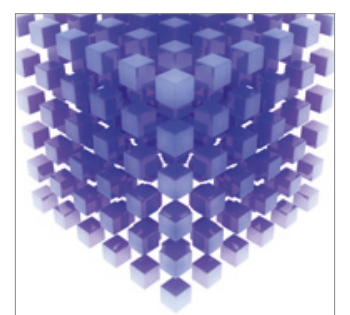

Mathematical Problems in Engineering
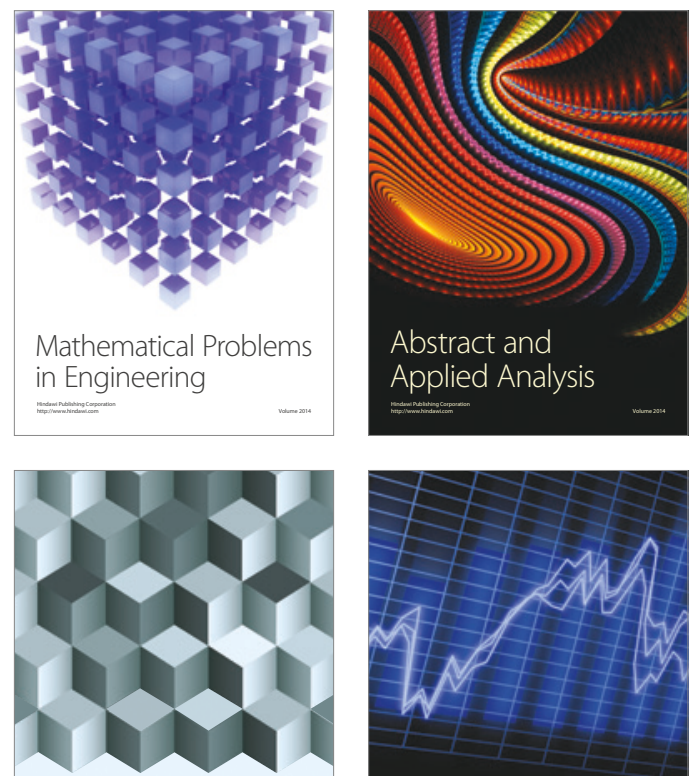

Journal of

Function Spaces

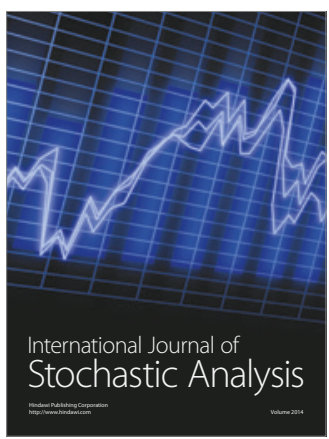

Probability and Statistics
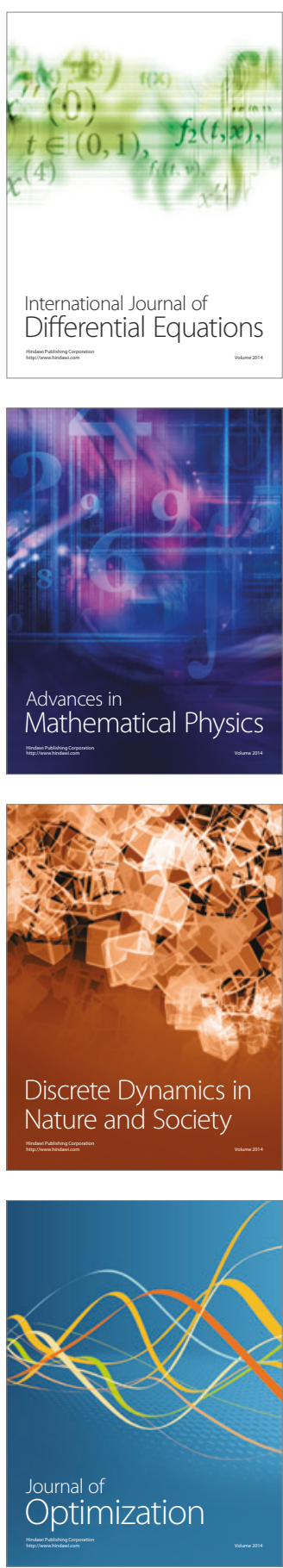